EPJ Web of Conferences 59, 13009 (2013)

DOI: $10.1051 /$ epjconf/20135913009

(C) Owned by the British Crown, published by EDP Sciences, 2013

\title{
Modelling Thomson scattering for systems with non-equilibrium electron distributions
}

\author{
D.A. Chapman ${ }^{1,2, a}$, J. Vorberger ${ }^{2}$, K. Wünsch ${ }^{2}$ and D.O. Gericke ${ }^{2}$ \\ ${ }^{1}$ Plasma Physics Department, AWE plc, Aldermaston RG7 4PR, UK \\ ${ }^{2}$ CFSA, Department of Physics, University of Warwick, Coventry CV4 7AL, UK
}

\begin{abstract}
We investigate the effect of non-equilibrium electron distributions in the analysis of Thomson scattering for a range of conditions of interest to inertial confinement fusion experiments. Firstly, a generalised one-component model based on quantum statistical theory is given in the random phase approximation (RPA). The Chihara expression for electron-ion plasmas is then adapted to include the new non-equilibrium electron physics. The theoretical scattering spectra for both diffuse and dense plasmas in which non-equilibrium electron distributions are expected to arise are considered. We find that such distributions strongly influence the spectra and are hence an important consideration for accurately determining the plasma conditions.
\end{abstract}

\section{INTRODUCTION}

Thomson scattering (TS) is being developed as a diagnostic for inertial confinement fusion (ICF) experiments, which can cover a wide range of conditions. Weakly coupled plasmas are created from the ablated hohlraum wall and the fill gas, whereas high-density states with temperatures ranging from $1 \mathrm{eV}-10 \mathrm{keV}$ occur during the compression and burn stages. Current theoretical approaches for modelling the scattered spectrum are based on the equilibrium fluctuation-dissipation theorem. However, it is well known that the electrons are not always in equilibrium, e.g. for measurements made on time scales that are short compared to the relaxation time or in strongly driven systems, whereby a drive or probe pulse continuously perturbs the plasma $[1,2]$. Under these circumstances a more general description is required to model the TS signal.

The scattered power spectrum measured in TS experiments is given in terms of the dynamic structure factor (DSF) $S_{e e}^{\text {tot }}(\mathbf{q}, \omega)$, which is defined as the Fourier transform of the density-fluctuation autocorrelation function and describes the microscopic correlations between all the electrons in space and time [3]. A completely general form of the DSF can be written in terms of the correlation function for the density response of the fully coupled system, which contains all non-ideal effects such as strong coupling and partial ionization. Unfortunately no such general solution currently exists. However, the electrons are often weakly coupled for ICF conditions due to either high temperature or degeneracy, and thus the random phase approximation (RPA) is often sufficient. The basic conditions of the sample under study, in particular the mean electron density and temperature, can then be inferred by fitting the electronic response to experimental data.

\footnotetext{
${ }^{a}$ e-mail: david.chapman@awe.co.uk
}

This is an Open Access article distributed under the terms of the Creative Commons Attribution License 2.0, which permits unrestricted use, distribution, and reproduction in any medium, provided the original work is properly cited. 


\section{EPJ Web of Conferences}

\section{ELECTRONIC STRUCTURE IN NON-EQUILIBRIUM PLASMAS}

The fully coupled dielectric response of an electron gas can be derived for an arbitrary distribution function within a quantum statistical framework using non-equilibrium Green's functions [4]. Specifically, the DSF is defined in terms of the correlation function for the density response, which in a local approximation is related to the polarisation function via

$$
S_{e e}(\mathbf{q}, \omega ; t)=\frac{i \hbar}{2 \pi n_{e}(t)} \chi_{e e}^{>}(\mathbf{q}, \omega ; t)=\frac{i \hbar}{2 \pi n_{e}(t)} \frac{\Pi_{e e}^{>}(\mathbf{q}, \omega ; t)}{\left|1-V_{e e}(q) \Pi_{e e}^{R}(\mathbf{q}, \omega ; t)\right|^{2}},
$$

for a one-component electron plasma. In the RPA the polarisation bubble is constructed from bare propagators, i.e. undressed Green's functions, in which no self-energy or exchange effects are considered. The time-ordered polarisation function is then simply the product of two singleparticle Green's functions. Using the Kadanoff-Baym ansatz in the quasi-particle picture the resulting expressions can be shown to be

$$
\begin{gathered}
\Pi_{e e}^{>}(\mathbf{q}, \omega ; t)=-2 i \int \frac{\mathrm{d}^{3} \mathbf{p}}{(2 \pi \hbar)^{3}} 2 \pi \delta\left(E_{e}(\mathbf{p}+\mathbf{q})-E_{e}(\mathbf{p})-\hbar \omega\right)\left[1-f_{e}(\mathbf{p}+\mathbf{q} ; t)\right] f_{e}(\mathbf{p} ; t), \\
\Pi_{e e}^{R}(\mathbf{q}, \omega ; t)=2 \int \frac{\mathrm{d}^{3} \mathbf{p}}{(2 \pi \hbar)^{3}} \frac{f_{e}(\mathbf{p}+\mathbf{q} ; t)-f_{e}(\mathbf{p} ; t)}{E_{e}(\mathbf{p}+\mathbf{q})-E_{e}(\mathbf{p})-(\hbar \omega+i 0)},
\end{gathered}
$$

where $f_{e}(\mathbf{p} ; t)$ and $E_{e}(\mathbf{p})=p^{2} / 2 m_{e}$ are the time-dependent distribution function and kinetic energy of the electrons respectively. Furthermore, Eq. (1) may be equivalently expressed in terms of the structure of the non-interacting system $2 \pi n_{e} S_{e e}^{0}(\mathbf{q}, \omega)=i \hbar \Pi_{e e}^{>}(\mathbf{q}, \omega)$

$$
S_{e e}(\mathbf{q}, \omega ; t) \stackrel{\mathrm{RPA}}{=} \frac{S_{e e}^{0}(\mathbf{q}, \omega ; t)}{|\varepsilon(\mathbf{q}, \omega ; t)|^{2}} .
$$

The scattering process may be qualitatively understood by considering the Feynman diagram for s-wave Compton scattering, averaging over the distribution of initial electron momenta with the blocking factor $f_{e}(\mathbf{p}+\mathbf{q} ; t)$ for the outgoing states, and including the effects of dynamic screening via the dielectric function $\varepsilon(\mathbf{q}, \omega ; t)=1-V_{e e}(q) \Pi_{e e}^{R}(\mathbf{q}, \omega ; t)$ [5]. Finally, taking the equilibrium Fermi-Dirac distribution function for the electrons and inserting into Eqs. (1)-(3) then gives the familiar form of the fluctuation-dissipation theorem as expected

$$
S_{e e}(\mathbf{q}, \omega ; t)=\frac{\hbar}{\pi n_{e}} \frac{1}{1-e^{-\beta_{e} \hbar \omega}} \frac{\mathfrak{I m} \Pi_{e e}^{R}(\mathbf{q}, \omega ; t)}{|\varepsilon(\mathbf{q}, \omega ; t)|^{2}} .
$$

The theoretical scattering spectrum for a two-component plasma of electrons and ions can be constructed via the approach of Chihara [6], in which the dynamic response is separated into a high-frequency plasmon term, a low-frequency acoustic term and an inelastic bound-free term. For weakly coupled electrons the plasmon term can be taken in RPA using Eq. (5). The non-equilibrium physics must also be included self-consistently in low-frequency response by means of a modified screening form factor, $\rho_{s c}(q ; t)$. In linear response approximation for small momenta this is simply [7]

$$
\begin{gathered}
\rho_{s c}(q ; t)=Z_{f} V_{e e}(q) \frac{\Pi_{e e}^{R}(\mathbf{q}, 0 ; t)}{\varepsilon(\mathbf{q}, 0 ; t)} \stackrel{q \rightarrow 0}{=} Z_{f} \frac{\kappa_{e}^{2}(t)}{\kappa_{e}^{2}(t)+(q / \hbar)^{2}}, \\
\kappa_{e}^{2}(t)=\frac{e^{2}}{\varepsilon_{0}} \int \frac{\mathrm{d}^{3} \mathbf{p}}{(2 \pi \hbar)^{3}} \frac{f_{e}(\mathbf{p} ; t)}{E_{e}(\mathbf{p})},
\end{gathered}
$$


for the number of free electrons per atom $Z_{f}$. The bound-free term may be neglected for low- $Z$ plasmas or for long wavelength probing radiation with insufficient energy to produce photoionization. In this work we focus only on the plasmon term in order to not confuse the effects of strong coupling and non-equilibrium physics in the low-frequency component of the spectrum.

\section{RESULTS}

In previous work $[5,8]$ we have shown that the spectrum of TS from strongly driven warm dense hydrogen probed with soft x-rays from the FLASH free electron laser (FEL) is significantly modified by non-equilibrium electron physics. In particular, the distribution function was taken from simulations of the FEL-target interaction using an atomic kinetics code, and a time-integrated treatment was shown to reproduce experimental data [2].

Here we consider two other common models for non-equilibrium electron distributions of relevance to TS under ICF conditions. Firstly, we consider collective TS from diffuse, high temperature plasmas created in long-pulse laser-driven targets probed with optical radiation [1]. In this case, strong inverse Bremsstrahlung heating from the probe beam leads to a steady-state distribution function with a roughly super-Gaussian bulk and a Maxwellian tail [9, 10]. Figure 1(a) shows the red-shifted plasmon data from Ref. [1] fitted with calculations using this modified super-Gaussian form compared to equilibrium calculations for different conditions. As expected, our model gives qualitatively similar results to those of Ref. [10] since the wave numbers probed are small and the degeneracy is low, so that quantum mechanical effects such as Compton recoil and Pauli blocking are not important.

It is clear that whilst an equilibrium calculation can reproduce the damping and shift of the plasmon, although the temperature and density differ by $\sim 20 \%$ to the best fit conditions, a robust fit cannot be obtained over the whole range of scattered wavelengths. On the other hand, the signal can be well fitted over the entire range when the non-equilibrium physics is considered. Furthermore, decreasing the index of the super-Gaussian both shifts and broadens the peak, demonstrating the sensitivity of the plasmon dispersion and damping to the distribution function. The errors introduced by an equilibrium analysis are therefore potentially substantial for subsequent calculations of interest to ICF, e.g. parametric and turbulent instability growth in hohlraums [11].

We have also previously considered the effect of a population of hot electrons on the spectrum of collective TS from isochorically heated Be probed with x-rays [12]. Due to the high density and low temperature of such states the model of Ref. [10] is insufficient as quantum effects are important, and a full quantum statistical treatment is needed. In this case, the plasma was considered to be in equilibrium due to the $\sim 0.5 \mathrm{~ns}$ delay between the pump and the probe. However, it is reasonable to expect that a quasithermal hot tail may persist due to the substantially longer equilibration rate of the hot electrons. Taking a simple two-temperature model for the distribution function $f_{e}(\mathbf{p}) \approx$ $\left(1-\phi_{h}\right) f_{c}\left(\mathbf{p}, T_{c}\right)+\phi_{h} f_{h}\left(\mathbf{p}, T_{h}\right)$, where the fraction of hot electrons is $\phi_{h}=n_{h} / n_{e}$ and the cold and hot components are represented by Fermi-Dirac distributions at different temperatures, the detailed balance in particular was shown to be significantly affected [5].

Here we investigate the effect of a similar hot tail on the noncollective spectrum for the same conditions by increasing the scattering angle to $\theta=130^{\circ}$. Figure 1 (b) shows that the noncollective spectrum is not as sensitive to the hot component, although increasing $\phi_{h}$ both noticeably alters the amplitude and the wings of the Compton peak. Increasing the temperature of the hot tail reduces the prominence of the wings, but further decreases the amplitude of the main peak. It is also interesting to note that for a modest tail (with a large fraction and relatively low temperature) the spectrum appears to develop a small peak (see inset) on the blue-shifted side of the probe, shown by the vertical dashed line, despite the scattering parameter $\alpha=\hbar \kappa_{e} / q$ being slightly reduced compared to the equilibrium conditions. The main effect of the hot tail on the backscattered spectrum is therefore on the amplitude of the Compton peak. This is potentially important as the charge state required to balance the relative strengths of the inelastic and elastic scattering signals may be in error relative 

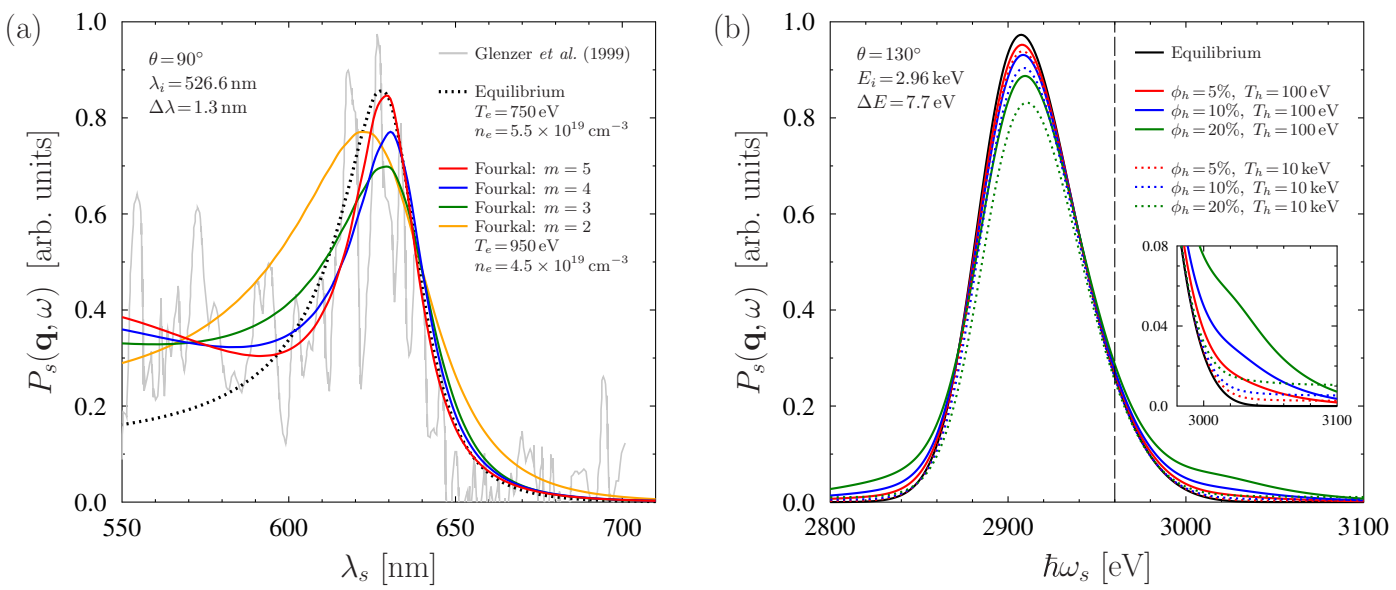

Figure 1. (a) Theoretical fit to plasmon data (noisy grey curve) in laser-produced Au plasma using the distribution of Fourkal et al. [10] for $m=5$ (red curve), $m=4$ (blue curve), $m=3$ (green curve) and $m=2$, i.e. Maxwellian, (orange). The best fit constrained by equilibrium (black curve) is shown for comparison. (b) Theoretical power spectra for warm dense Be at $T_{e}=12 \mathrm{eV}$ and $n_{e}=3 \times 10^{23} \mathrm{~cm}^{-3}$ including a hot tail with $T_{h}=100 \mathrm{eV}$ (solid curves) and $T_{h}=10 \mathrm{keV}$ (dashed curves), and various weights $\phi_{h} ; 5 \%$ (red curve), $10 \%$ (blue curve) and $20 \%$ (green curve) compared to an equilibrium calculation (black curve) [12].

to equilibrium. Specifically, a larger $Z_{f}$ would be need to be invoked, which could be attributed to enhanced impact ionization due to the hot electrons.

\section{CONCLUSIONS}

We have developed a model for TS based on quantum statistics which may be applied to a wide range of experiments of interest to ICF. We have produced theoretical fits to data from optically probed laserproduced Au plasmas and have shown qualitative agreement with previous work; the fit to the spectrum is essentially unmodified since quantum mechanical effects such as Compton recoil or Pauli blocking are not important under these conditions. We have also applied our model to investigating the effect of a hot tail component on the spectrum of $\mathrm{x}$-rays scattered by warm dense $\mathrm{Be}$ in the noncollective regime. We found that the spectrum is not as noticeably modified as that of the forward scattered spectrum, although a reduction in the height of the Compton feature was observed. Consequently, the charge state inferred from the relative amplitudes of the elastic and inelastic peaks may be in error if the plasma is not in equilibrium. We conclude that a rigorous treatment of non-equilibrium physics in modelling TS is an important consideration for plasmas with ICF-relevent conditions.

\section{References}

[1] S.H. Glenzer et al., Phys. Plasmas 6, 2117 (1999)

[2] R.R. Fäustlin et al., Phys. Rev. Lett. 104, 125002 (2010)

[3] J. Sheffield, Plasma scattering of electromagnetic radiation (Academic Press, London 1975)

[4] D. Kremp, M. Schlanges, and W.-D. Kraeft, Quantum statistics of nonideal plasmas (Springer, Berlin 2006)

[5] D.A. Chapman and D.O.Gericke, Phys. Rev. Lett. 107, 165004 (2011)

[6] J. Chihara, J. Phys. F: Met. Phys. 17, 295 (1987)

[7] D.O. Gericke et al., Phys. Rev. E 81, 065401(R) (2010) 


\section{IFSA 2011}

[8] D.A. Chapman et al., High Energy Density Physics 8, 175 (2012)

[9] S.H. Glenzer et al., J. Quant. Spectrosc. Radiat. Transfer 65, 253 (2000)

[10] E. Fourkal et al., Phys. Plasmas 8, 550 (2001)

[11] S.H. Glenzer et al., Phys. Rev. Lett 88, 235002 (2002)

[12] S.H. Glenzer et al., Phys. Rev. Lett. 98, 065002 (2007) 